\title{
Evaluation of a novel portable capacitive ECG system in the clinical practice for a fast and simple ECG assessment in patients presenting with chest pain: FIDET (Fast Infarction Diagnosis ECG Trial)
}

\author{
Eva C. L. Rasenack • Martin Oehler • Albrecht Elsässer • \\ Meinhard Schilling • Lars S. Maier
}

Received: 22 June 2012/ Accepted: 18 September 2012/Published online: 29 September 2012

(C) The Author(s) 2012. This article is published with open access at Springerlink.com

\begin{abstract}
Background Electrocardiogram (ECG) assessment plays a crucial role in patients presenting with chest pain and suspected acute coronary syndrome (ACS). In a pilot study, we previously evaluated a capacitive ECG system (cECG) as a novel ECG technique for a fast and simple ECG assessment in patients with ST-elevation myocardial infarction (STEMI). In a next step, the sensitivity and specificity of this novel ECG technique have to be assessed in patients with ACS.

Hypothesis The Fast Infarction Diagnosis ECG Trial (FIDET) is a prospective, bi-center, observer-blinded noninferiority study to evaluate the cECG compared to the conventional ECG (kECG) in the clinical practice for ECG assessment in consecutive patients presenting with suspected ACS.

Methods In 250 patients who were admitted to the hospital, because of an ACS [including STEMI and non-STelevation acute coronary syndrome (NSTE-ACS)], both a
\end{abstract}

E. C. L. Rasenack · L. S. Maier $(\bowtie)$

Department of Cardiology and Pneumology/Heart Center,

Georg-August-University Göttingen, Robert-Koch-Str. 40,

37075 Göttingen, Germany

e-mail: 1maier@med.uni-goettingen.de

M. Oehler

Capical GmbH, Braunschweig, Germany

A. Elsässer

Clinic for Cardiology/Heart Center Oldenburg,

Oldenburg, Germany

M. Schilling

Institute of Electrical Measurement and Fundamental

Electrical Engineering, TU, Braunschweig, Germany
kECG and a cECG recording were performed within a time lag of less than $10 \mathrm{~min}$.

End points The primary end point will be sensitivity and specificity of the cECG compared to the kECG in diagnosing a STEMI with a margin of noninferiority of $7.5 \%$. Secondary end points include sensitivity and specificity of the cECG compared to the KECG in diagnosing an NSTEACS, safety of the cECG system (adverse event, serious adverse event and suspected unexpected serious adverse reaction), parameters of the ECG measurement (PQ-interval, QT-interval, ST-amplitude and heart rate) and measurement duration of the two methods.

Conclusion FIDET is designed as a noninferiority study to show that a novel cECG system is suitable for the diagnosis of myocardial infarction in the clinical context and might even have benefits, for example by offering a faster and easier ECG assessment.

Keywords Capacitive ECG system - Capacitive electrodes - Myocardial infarction - Acute coronary syndrome $\cdot$ Clinical cardiology $\cdot$ ECG assessment

$\begin{array}{ll}\text { Abbreviations } \\ \text { ACS } & \text { Acute coronary syndrome } \\ \text { AE } & \text { Adverse event } \\ \text { cECG } & \text { Capacitive ECG system } \\ \text { CPU } & \text { Chest pain unit } \\ \text { eCRF } & \text { Electronic case report form } \\ \text { ECG } & \text { Electrocardiogram } \\ \text { ER } & \text { Emergency room } \\ \text { ICD } & \text { Implantable cardioverter defibrillator } \\ \text { kECG } & \text { Conventional (12-lead) ECG } \\ \text { MAL } & \text { Midaxillary line } \\ \text { MI } & \text { Myocardial infarction } \\ \text { NSTE-ACS } & \text { Non-ST-elevation acute coronary syndrome }\end{array}$




$\begin{array}{ll}\text { NSTEMI } & \begin{array}{l}\text { Non-ST-elevation myocardial } \\ \text { infarction }\end{array} \\ \text { PM } & \text { Pacemaker } \\ \text { SAE } & \begin{array}{l}\text { Serious adverse event } \\ \text { STEMI }\end{array} \\ \text { ST-elevation myocardial infarction } \\ \text { SUSAR } & \begin{array}{l}\text { Suspected unexpected serious } \\ \text { adverse reaction }\end{array}\end{array}$

\section{Introduction}

In 1902, Willem Einthoven published his first recordings of the heart from his "string galvanometer" which he named "elektrocardiogramm" [1]. In the past century, the electrocardiogram (ECG) became one of the most frequently used diagnostic devices all over the world and plays a crucial role in the diagnosis of acute myocardial infarction (MI) $[2,3]$. This is especially important in the pre-hospital phase, where an early diagnosis of an ST-elevation myocardial infarction (STEMI) sets the course for the rapid initiation of reperfusion therapy [4].

A so-called capacitive ECG system (cECG) is a novel technical innovation, which could be a useful diagnostic tool for a fast and simple ECG assessment. Unlike the conventional 12-lead ECG (kECG), there is no need to fix the electrodes to the skin and no need of electrolyte gel. This might be very useful in an emergency situation when a fast diagnosis is especially important, e.g., in patients presenting with an acute coronary syndrome (ACS). In hemodynamically unstable patients admitted to the hospital with shock or pre-shock, the adhesion of the conventional electrodes to the wet skin sometimes is hardly possible. In this situation, the application of a cECG system could be beneficial as well.

In a proof of concept pilot study that was performed between March 2009 and May 2010, we could show that it was possible to identify a STEMI with the cECG in clinical practice [6]. In 66 patients admitted to the hospital, because of a STEMI a cECG was performed and compared to a kECG of the same patient. During the course of the study, the cECG was modified, because the initially used ECG system was able to detect an anterior MI without a problem, but did not identify an inferior MI sufficiently. After adding an additional electrode further away from the electrodes on the chest at the lower back of the patient, STelevations in the inferior leads II, III and aVF could be assessed appropriately as well. After these first promising clinical results, further studies are needed to analyze the sensitivity and specificity of the novel innovative ECG technique.
Table 1 Inclusion and exclusion criteria

\begin{tabular}{ll}
\hline Inclusion criteria & Exclusion criteria \\
\hline $\begin{array}{l}\text { Male and female patients } \\
\geq 18 \text { years }\end{array}$ & Pregnant or breastfeeding women \\
$\begin{array}{l}\text { Patients presenting with chest } \\
\text { pain and suspected ACS } \\
\text { (acute coronary syndrome) }\end{array}$ & $\begin{array}{c}\text { Patients carrying a pacemaker } \\
\text { (PM) or implantable } \\
\text { cardioverter defibrillator (ICD) }\end{array}$ \\
$\begin{array}{l}\text { Signed written informed consent } \\
\text { Bundle-branch block visible in } \\
\text { the ECG (except for a new left } \\
\text { bundle-branch block) }\end{array}$ \\
$\begin{array}{c}\text { A 12-lead ECG is routinely } \\
\text { recorded in the hospital on } \\
\text { admission of the patient }\end{array}$ & $\begin{array}{c}\text { Cardiogenic shock } \\
\text { Patients who recently underwent } \\
\text { an operation at the chest }\end{array}$ \\
\hline
\end{tabular}

\section{Methods}

Study design

The FIDET study is a prospective, bi-center, observerblinded, single-arm noninferiority study, where the participant is the control group at the same time (protocol ID 20110819-MO, EUDAMED-No. CIV-11-08-002121). Our intention is to evaluate the cECG compared to the kECG in the clinical practice for ECG assessment in consecutive patients presenting with ACS with regard to reliability and speed. The trial was funded by Capical $\mathrm{GmbH}$, Braunschweig, Germany. It was approved by the ethics committee of the University Medical Center Göttingen as the leading ethics committee. As the measurements were carried out in patients who were in a potentially unstable state, where no time should be wasted, we got permission to obtain written informed consent after the ECG assessments.

\section{Conducting patient selection and study}

The duration of the study is supposed to be very short. Consecutive patients presenting to the hospital with ACS will be screened for inclusion into the study. Taking the different entities of ACS together (STEMI, NSTE-ACS), measurements will presumably be conducted in the emergency room (ER), the cardiac catheterization laboratory (before reperfusion therapy is started) and the chest pain unit (CPU). Inclusion and exclusion criteria are listed in Table 1. If a patient fulfills the inclusion and exclusion criteria, both a cECG and a kECG will be performed with a time lag of less than 10 min (flowchart of the protocol, Fig. 1). We choose this short interval to make the different ECG assessments as comparable as possible. There will be neither any further examinations nor a follow-up, but some of the patients' personal data will be collected in the electronic case report forms (eCRF) for further analysis. 


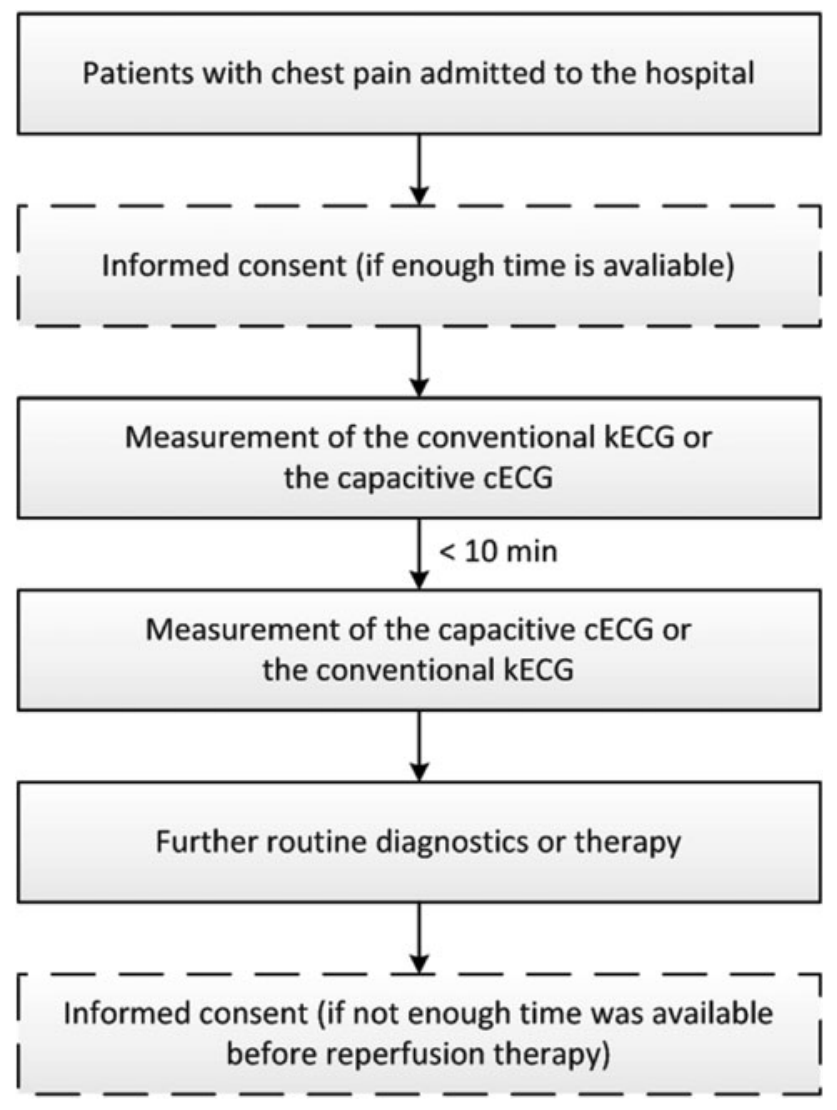

Fig. 1 Flowchart of the study protocol

We intend to include 250 patients. An interim safety analysis will be performed after recruitment of the first 100 patients.

Medical device (capacitive ECG system) and measuring procedure

The used cECG system is shown in Fig. 2; it consists of a sensor array with 25 flexible mounted capacitive electrodes integrated into a $\sim 20 \times 20 \mathrm{~cm}$ round mainframe and another 7 electrodes on the additional probe connected via cable to the mainframe.

While the measuring principle of the cECG and the kECG is the same, both detect potential differences during the electric heart action and the particular sensor (capacitive or galvanic electrode) works in a different mode. Capacitive electrodes, among other things for ECG measurements, were first described in the 1960s [7-9]. In contrast to the conventional galvanic electrodes that require an electrolyte gel for enabling a certain conductivity for measuring a bioelectric signal, they are isolated from the body and provide a capacitive coupling between the skin and a metallic face inside the sensor. The signal is injected in a capacitive way with high impedance, as the electrode

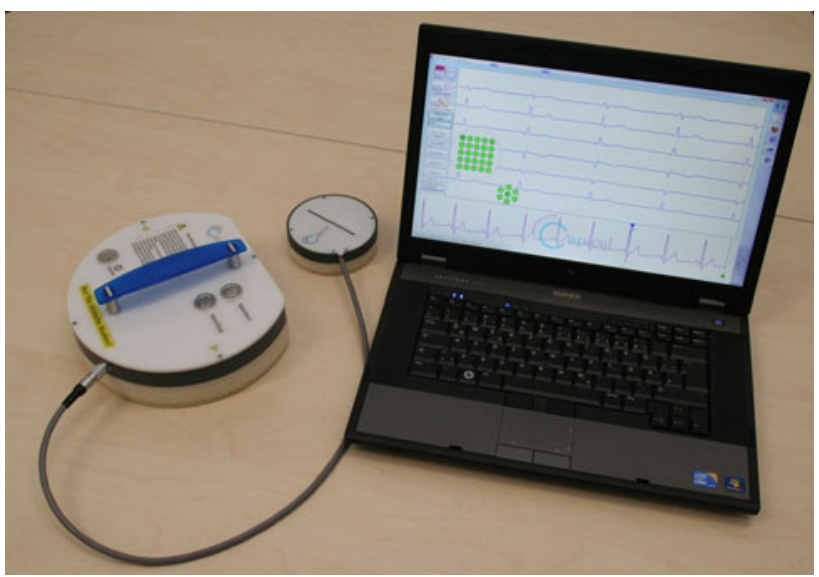

Fig. 2 The cECG device used consists of a sensor array with 25 flexible mounted capacitive electrodes integrated into a $\sim 20 \times 20 \mathrm{~cm}$ round mainframe and another 7 electrodes on the additional probe connected via cable to the mainframe. The recording is automatically sent to the laptop via Bluetooth connection

detects electric displacement currents that are caused by the changing potential distribution during the heartbeat. For this purpose, a special amplifier has to be connected to the metallic face.

With this configuration, it is possible to obtain an ECG recording with the cECG that gives exactly the same information as the kECG when the electrodes are placed at the same position, and comparable results if the capacitive electrodes are placed in an array like the cECG system used in this and previous studies [5, 10]. After modification of the cECG system in the course of our previous pilot study [6], the current clinical trial will be conducted with model number 2.0 and the software version 1.0.

For ECG assessment, the system is placed on the patient's undressed chest, the mainframe central on the chest in the range of the third intercostal space and the additional probe on the left side of the patient in the range of the fifth intercostal space in the midaxillary line (MAL) with the upper electrode on the Wilson V6 position (Fig. 3). The ECG recording is then controlled and saved by the mainframe, allowing a fast and simple handling. Once a recording is saved, it is automatically sent to the laptop via Bluetooth connection, where a further processing of the data is possible.

End points

The FIDET study will include the following end points:

1. Primary end point: sensitivity and specificity of the cECG in the detection of a STEMI compared to the international gold standard (kECG)

2. Secondary end points 

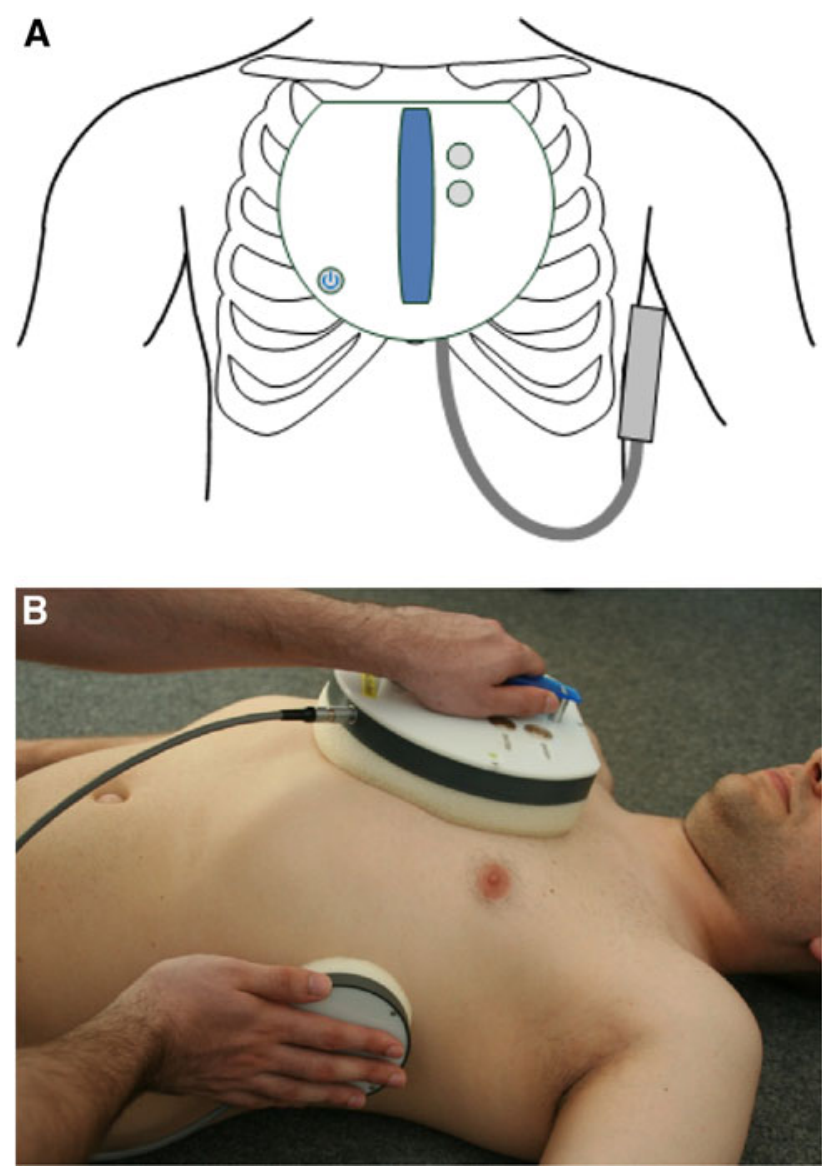

Fig. 3 Correct position of the cECG. a Schematic position of the cECG device on the chest and $\mathbf{b}$ demonstration of an ECG assessment

a. sensitivity and specificity of the cECG in diagnosing an NSTEMI compared to the gold standard (kECG, biomarker)

b. safety of the cECG system; documented by adverse events (AE), serious adverse events (SAE), occurrences, undesirable effects and serious undesirable effects of the product

c. different parameters of the ECG measurement (PQ-interval, QT-interval, ST-amplitude and heart rate)

d. measurement duration of the two different methods (kECG vs. cECG)

e. diagnostic information of space-resolved ECG data of all channels of the cECG via temporal and local analysis in comparison to the $\mathrm{kECG}$ as gold standard

Analysis and statistical considerations

The collected data will be acquired in an electronic CRF. The study database will be created by using the software secuTrail $^{\odot}$. As mentioned above, the FIDET study is designed as a prospective, bi-center, observer-blinded, single-arm noninferiority study to prove the suitability of the cECG for the diagnosis of MI in the clinical context. The cECG will be compared directly with the 12-lead ECG as the current gold standard for the diagnosis of MI. For analysis, evaluation of a 12-lead ECG by an investigator of one center will be compared to the cECG of the same patient evaluated by an investigator of the other study site. As the study is observer blinded, the evaluation of the cECG will be carried out without knowing the result of the kECG; therefore, there is no need for a control group.

The sensitivity and specificity of the cECG in detecting a STEMI compared to the gold standard (kECG) will be determined using a fourfold table and $95 \%$ confidence intervals will be calculated. A $p$ value of $<0.05$ will be considered as statistically significant. The sensitivity of the cECG in STEMI detection was estimated to be $92.5 \%$ based on the data from the prior study. Therefore, 84 patients with STEMI are needed to stay within the $95 \%$ confidence interval, choosing a $7.5 \%$ tolerance level. Corresponding results hold true for specificity. Based on an expected STEMI prevalence of $35 \%$ in the patient population, a sample size for this study of approximately 250 patients should be adequate to sufficiently match the requirements for the confidence interval calculation regarding sensitivity and specificity.

\section{Discussion}

One of the most common emergency situations is a patient presenting with chest pain. Unless proven otherwise, an ACS is suspected that requires a rapid diagnosis to initiate further therapy due to the impaired prognosis for patients with a possible MI. The 12-lead ECG is the most important diagnostic tool in this setting to distinguish between the different diseases involving the coronary arteries STEMI, NSTEMI or unstable angina (the latter two are newly called NSTE-ACS) [11]. In case of STEMI, a reperfusion therapy is indicated as soon as possible [4, 12].

Thus, new ECG techniques that offer a faster and easier ECG assessment are developed [13]. A portable, so-called cECG system based on capacitive electrodes permits a measurement of ECG signals without fixing the electrodes on the patient's skin or using electrolyte gel. To show that the cECG is a promising diagnostic tool in the clinical environment, we performed a pilot study in 66 patients admitted to the hospital with a STEMI. After modification of the cECG, we were able to detect both anterior and inferior MI. To analyze the sensitivity and specificity and further optimize the cECG system, the FIDET study is planned as a noninferiority study to prove that the innovative ECG technique is suitable to diagnose MI in the clinical context. A noninferiority margin of $7.5 \%$ was 
allowed. Therefore, a kECG as well as a cECG will be performed in consecutive patients admitted to the hospital with chest pain, thereby allowing a direct comparison between the two types of ECG examinations. A total of 250 patients will be included. The primary end point of the study will be sensitivity and specificity of the cECG compared to the kECG in diagnosing a STEMI. The primary end point is defined as sensitivity and specificity for STEMI diagnosis of the capacitive ECG compared to the gold standard 12-channel ECG. Per definition, the sensitivity and specificity of the 12-channel ECG is $100 \%$. Further, we will analyze the results of the coronary angiography of the STEMI patients to validate the sensitivity of the 12-channel ECG. This is not possible for specificity, because not all 250 patients underwent a coronary angiography. In a third step, the 12-channel ECG and the capacitive ECG will be compared to enzyme elevations. Basically, the treatment for the individual patient is very short and simple and a follow-up is not planned.

If the cECG can demonstrate its ability to reliably diagnose an MI, it might be a quite useful diagnostic device in hospital and especially in the pre-hospital phase, where a rapid diagnosis is required [14]. An integration of the cECG system into the patient monitor used in the emergency medical service, for example, would make the assessment of ECG and the subsequent transmission to a PCI center by the cardiologist on duty very fast, easy and straightforward and may even further improve outcome [15].

In conclusion, a rapid diagnosis in patients presenting with chest pain is of utmost importance. The so-called capacitive ECG system, a novel ECG technique with capacitive electrodes, was able to identify STEMIs in a pilot study. The FIDET was designed as a noninferiority study to investigate sensitivity and specificity of cECG and thus prove that it is suitable for diagnosing MI in clinical practice and might even have benefits by offering a particularly fast and simple ECG assessment.

Acknowledgments Dr. Maier is funded by the DFG through a Heisenberg grant (MA1982/4-1), a Klinische Forschergruppe grant (MA1982/2-2), Teilprojekt A03 of the Sonderforschungsbereich 1002, as well as the Fondation Leducq Transatlantic Network of Excellence. This study was funded by an investigative grant by Capical GmbH, Braunschweig, Germany.

Open Access This article is distributed under the terms of the Creative Commons Attribution License which permits any use, distribution, and reproduction in any medium, provided the original author(s) and the source are credited.

\section{References}

1. Einthoven W (1902) Galvanometrische registratie van het menselijk electrocardiogram. In: Herinneringsbundel Rosenstern
SS (ed) Galvanometric registration of the human electrogram. Eduard Ijdo, Leiden, pp 101-106

2. Van de Werf F, Bax J, Betriu A, Blomstrom-Lundqvist C, Crea F, Falk V, Filippatos G, Fox K, Huber K, Kastrati A, Rosengren A, Steg PG, Tubaro M, Verheugt F, Weidinger F, Weis M, ESC Committee for Practice Guidelines (CPG) (2008) Management of acute myocardial infarction in patients presenting with persistent ST-segment elevation: the Task Force on the Management of STSegment Elevation Acute Myocardial Infarction of the European Society of Cardiology. Eur Heart J 29:2909-2945

3. Antman EM, Anbe DT, Armstrong PW, Bates ER, Green LA, Hand M, Hochman JS, Krumholz HM, Kushner FG, Lamas GA, Mullany CJ, Ornato JP, Pearle DL, Sloan MA, Smith SC Jr, Alpert JS, Anderson JL, Faxon DP, Fuster V, Gibbons RJ, Gregoratos G, Halperin JL, Hiratzka LF, Hunt SA, Jacobs AK, American College of Cardiology/American Heart Association Task Force on Practice Guidelines (Writing Committee to Revise the 1999 Guidelines for the Management of Patients With Acute Myocardial Infarction) (2004) ACC/AHA guidelines for the management of patients with ST-elevation myocardial infarctionexecutive summary: a report of the American College of Cardiology/American Heart Association Task Force on Practice Guidelines (writing committee to revise the 1999 guidelines for the management of patients with acute myocardial infarction). Circulation 110:588-636

4. Diercks DB, Kontos MC, Chen AY, Pollack CV Jr, Wiviott SD, Rumsfeld JS, Magid DJ, Gibler WB, Cannon CP, Peterson ED, Roe MT (2009) Utilization and impact of pre-hospital electrocardiograms for patients with acute ST-segment elevation myocardial infarction: data from the NCDR (National Cardiovascular Data Registry) ACTION (Acute Coronary Treatment and Intervention Outcomes Network) Registry. J Am Coll Cardiol 53:161-166

5. Oehler M, Ling V, Melhorn K, Schilling M (2008) A multichannel portable ECG system with capacitive sensors. Physiol Meas 29:783-793

6. Weil MB, Oehler M, Schilling M, Maier LS (2012) First clinical evaluation of a novel capacitive ECG system in patients with acute myocardial infarction. Clin Res Cardiol 101:165-174

7. Richardson PC (1967) The insulated electrode: a pasteless electrocardiographic technique. 20th Annual Conference on Engineering in Medicine and Biology, vol 9, p 15.7

8. Richardson PC, Coombs FK, Adams RM (1968) Some new electrode techniques for long-term physiologic monitoring. Aerosp Med 39:745-750

9. Lopez A Jr, Richardson PC (1969) Capacitive electrocardiographic and bioelectric electrodes. IEEE Trans Biomed Eng 16:99

10. Oehler M, Schilling M, Esperer HD (2009) Capacitive ECG system with direct access to standard leads and body surface potential mapping. Biomed Tech (Berl) 54:329-335

11. Hamm CW, Bassand JP, Agewall S, Bax J, Boersma E, Bueno H, Caso P, Dudek D, Gielen S, Huber K, Ohman M, Petrie MC, Sonntag F, Uva MS, Storey RF, Wijns W, Zahger D; ESC Committee for Practice Guidelines, Bax JJ, Auricchio A, Baumgartner H, Ceconi C, Dean V, Deaton C, Fagard R, FunckBrentano C, Hasdai D, Hoes A, Knuuti J, Kolh P, McDonagh T, Moulin C, Poldermans D, Popescu BA, Reiner Z, Sechtem U, Sirnes PA, Torbicki A, Vahanian A, Windecker S; Document Reviewers, Windecker S, Achenbach S, Badimon L, Bertrand M, Bøtker HE, Collet JP, Crea F, Danchin N, Falk E, Goudevenos J, Gulba D, Hambrecht R, Herrmann J, Kastrati A, Kjeldsen K, Kristensen SD, Lancellotti P, Mehilli J, Merkely B, Montalescot G, Neumann FJ, Neyses L, Perk J, Roffi M, Romeo F, Ruda M, Swahn E, Valgimigli M, Vrints CJ, Widimsky P (2011) ESC Guidelines for the management of acute coronary syndromes in 
patients presenting without persistent ST-segment elevation: the Task Force for the Management of Acute Coronary Syndromes (ACS) in patients presenting without persistent ST-segment elevation of the European Society of Cardiology (ESC). Eur Heart J 32: 2999-3054

12. Eitel I, Franke A, Schuler G, Thiele H (2010) ST-segment resolution and prognosis after facilitated versus primary percutaneous coronary intervention in acute myocardial infarction: a metaanalysis. Clin Res Cardiol 99:1-11

13. Tölg R, Zeymer U, Birkemeyer R, Wessely R, Eggebrecht H, Bocksch W, Schneider S, Richardt G, Hamm C (2012) Cardiogoniometry as a diagnostic tool in patients with acute coronary syndromes: results of the CGM@ACS trial. Clin Res Cardiol 101:727-736
14. Post F, Giannitsis E, Riemer T, Maier LS, Schmitt C, Schumacher B, Heusch G, Mudra H, Voigtländer T, Erbel R, Darius H, Katus H, Hamm C, Senges J, Gori T, Münzel T (2012) Pre- and early in-hospital procedures in patients with acute coronary syndromes: first results of the "German Chest Pain Unit Registry". Clin Res Cardiol [Epub ahead of print]

15. Scholz KH, Maier SKG, Jung J, Fleischmann C, Werner GS, Olbrich HG, Ahlersmann D, Keating FK, Jacobshagen C, Moehlis H, Hilgers R, Maier LS (2012) Reduction in treatment times through formalized data feedback results in improved prognosis in STEMI patients. JACC Cardiovasc Interv 5: $848-857$ 